\title{
LAND PRODUCTIVITY EVALUATION OF EDFU AREA, ASWAN, EGYPT, USING REMOTE SENSING AND GEOGRAPHIC INFORMATION SYSTEM TECHNIQUES
}

\author{
A. I. El-Desoky and Y. A. Sayed \\ Soils and Water Sci. Dept., Fac. Agric., Al-Azhar Univ., Assiut, Egypt. \\ Corresponding author [e-mail:yasser_soils@yahoo.com], \\ Received: Feb. 19, 2019 \\ Accepted: Feb. 26, 2019
}

\begin{abstract}
Due to horrific population explosion, that decreases the share of cultivated area in Egypt, more reclamation land must be situated. The present work aims to identify land productivity in the west part of Edfu city, Aswan Governorate using remote sensing and geographic information systems (GIS) techniques. The soils of the studied area belong to two land productivity classes according to the Riquier Land Productivity Index (RLPI). These classes are grade III (average) and grade IV (poor) representing 71.87 and $28.13 \%$ of the total area, respectively. The same soils belong to two land productivity classes according to the Land Productivity Spatial Model (LPSM) namely moderate productivity and low productivity representing 77.29 and $22.71 \%$ of the total investigated area, respectively. A significant correlation coefficient was observed between LPSM and RLPI models. These lands have moderate to very severe limitations that restrict their use for agriculture sector and require special conservation practices. These soils have fair to marginal productivity and recommended for producing forage crops and agro-forestry systems.
\end{abstract}

Key words: Land productivity, Remote sensing, GIS, Riquier Land Productivity Index, Edfu city.

\section{INTRODUCTION}

The most populous world exists in agricultural rural areas of developing countries. Therefore, agriculture sector remains the main activity to provide people with their need of food and fibers (Constanza et al., 1992; Pearce \& Warford, 1993 and Andzo-Bika \& Kamitewoko, 2004). The ancient cultivated area in Egypt occupied about $4 \%$ only from the total area represented by the Delta and Nile Valley that are the most suitable areas for sustainable agriculture. It is one of the oldest cultivated areas around the world, but is considered densely populated areas and surrounded by deserts (Zeydan, 2005). Agriculture is the most important sector in the sustainable development processes in Africa and the whole world (World Bank, 2007).
Agricultural productivity can be measured by the Total Productivity Factor (TPF) which is a calculating method that compare between agricultural inputs and output index (Fuglie et al., 2007). Land productivity is a total productivity associated with various factors such as parent material, geomorphologic units, climatology elements, and physiochemical soil characteristics, (Deng et al., 2011 and Zhou et al., 2012). The land productivity can be improved when farmers employ adopt improved soil environmentally friendly and management techniques. Assessment and monitoring of land productivity help in refining agricultural practices to maintain soil capacity for food production, fiber and commodity goods (Field, 2017 and Osuji \& Henri-Ukoha, 2017). 
Land productivity assessment is usually done directly or indirectly. The direct methods could be used experiments under certain climatic conditions with controlled administrative practices and conducted in the field or in greenhouses. Indirect methods depend on the development models to estimate land productivity rates. A well-known valuable and parametrical model was proposed as evaluation method for assessing and monitoring land productivity according to Riquier et al. (1970). This method provides one valuable index called land productivity index (LPI) that derived from soil properties related to vegetation. Repetition that manages our land resources is critical to the maintenance of land productivity to contribute significantly to the economy. It is also relevant to the livelihood of rural people (Schartzl and Anderson, 2006; FAO, 2007; Dengiz \& Sağlam, 2012 and Baskan et al., 2017).
The current research aims to assess the land productivity of Edfu area; Aswan, Egypt based on land resources, soil properties using remote sensing and geographic information system (GIS) techniques.

\section{MATERIALS AND METHODS}

Site description

The study area covers about $702 \mathrm{~km}^{2}$ in the west part of Edfu city, Aswan governorate between longitudes $32^{\circ} 35^{\prime}$ $39.11^{\prime \prime}$ and $32^{\circ} 49^{\prime} 45.44^{\prime \prime} \mathrm{E}$ and latitudes 24 ${ }^{\circ} 38^{\prime} 3.02^{\prime \prime}$ and $24^{\circ} 58^{\prime} 15.11^{\prime \prime} \mathrm{N}$ (Fig. 1). According to Egyptian Meteorological Authority (2011), the climatic regime is hot and characterized by normal winter and very hot arid summer typically called a desert climate. There is almost no rainfall during the year (about $1 \mathrm{~mm}$ of precipitation). The average annual temperature is $26.8{ }^{\circ} \mathrm{C}$ in Edfu. The studied area could be classified as Hyperthermic temperature regime and Torric soil moisture regime (Soil Survey Staff, 2014).

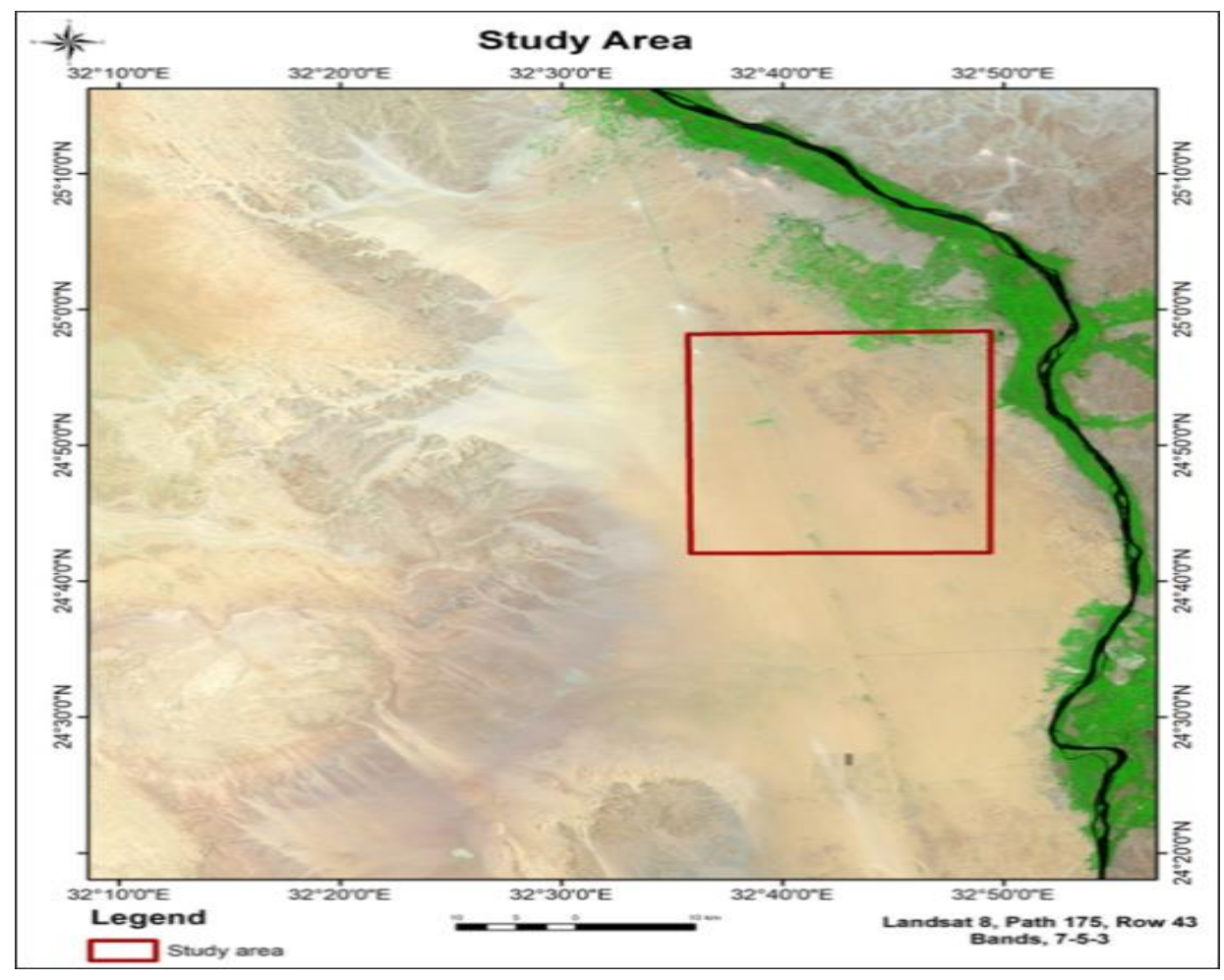

Fig. (1): Location map of the studied area. 
Digital image processing

Landsat 8 satellite image for the study area was taken during 2018 and corrected geometrically. Shuttle Radar Topography Mission (SRTM) images were used as the source data for digital elevation model of the study area (DEM). Image was draped over DEM to get the feel of natural three dimensions (3D) then for identifying and delineating the landforms of the study area the ENVI 5.1 software was implemented (ITT, 2017). The geomorphologic, and land productivity maps of the study area were layout, annotated, projected and finally produced using Arc GIS 10.2.2 software(ESRI, 2014).

\section{Field and laboratory investigations}

Field studies and ground proofing's were carried out based on the geomorphological map of the study area. Morphological descriptions of twentythree soil profiles representing different geomorphological units were carried out according to Burt (2014). Soil samples were collected and laboratory analyzed following the standard methods of Burt (2014).

\section{Riquier Land Productivity Index (RLPI)}

The Riquier Land Productivity Index (RLPI) of the various mapping units in the study area is estimated using the model produced by Riquier et al. (1970). Soil depth, organic matter, texture, soluble salt, soil reaction $(\mathrm{pH})$ of the surface layer, cation exchange capacity, drainage, slope, moisture content, and mineral reserve were used as a multiplied to outputs of the Riquier Land Productivity Index (RLPI) as follows:

Riquier Land Productivity Index (RLPI) $=(M / 100) \times(D / 100) \times(E / 100) \times(T / 100) \times$ $(\mathrm{S} / 100) \times(0 / 100) \times(C / 100) \times(R / 100) \times 100$.

Where,

RLPI = Riquier Land Productivity Index

$M=$ Moisture availability

$D=$ Drainage

$\mathrm{E}=$ Depth

$T=$ Texture

$S=$ Soluble salt concentration

$\mathrm{O}=$ Organic matter

C = Cation exchange capacity

$\mathbf{R}=$ Mineral reserves.

Land productivity classes and matching RLPI rates are shown in Table 1.

\section{RESULTS AND DISCUSSION}

Geomorphology of the studied area

Geomorphologic features could be identified throughout interpreting satellite image and DTM which are considered as advanced techniques. The basic advantages of satellite image afford the reality to the ground observation. Satellite image interpretation and field study indicated that, the investigated area includes four main geomorphologic units and two subunits (five landforms) as the following: 1) River terraces (with two subunits i.e. high and low River terraces); 2) Overflow basin; 3) Decantation basin; 4) Dry valleys. These geomorphologic unites are presented in Fig. (2) and Table (2).

Table (1). Land productivity classes and the matching RLPI rates.

\begin{tabular}{|c|c|c|c|}
\hline No. & Productivity Class & Symbol & RLPI rates \\
\hline 1 & Excellent & I & $65-100$ \\
\hline 2 & Good & II & $35-64$ \\
\hline 3 & Average & III & $20-34$ \\
\hline 4 & Poor & IV & $8-19$ \\
\hline 5 & Extremely poor to nil & V & $0-7$ \\
\hline
\end{tabular}




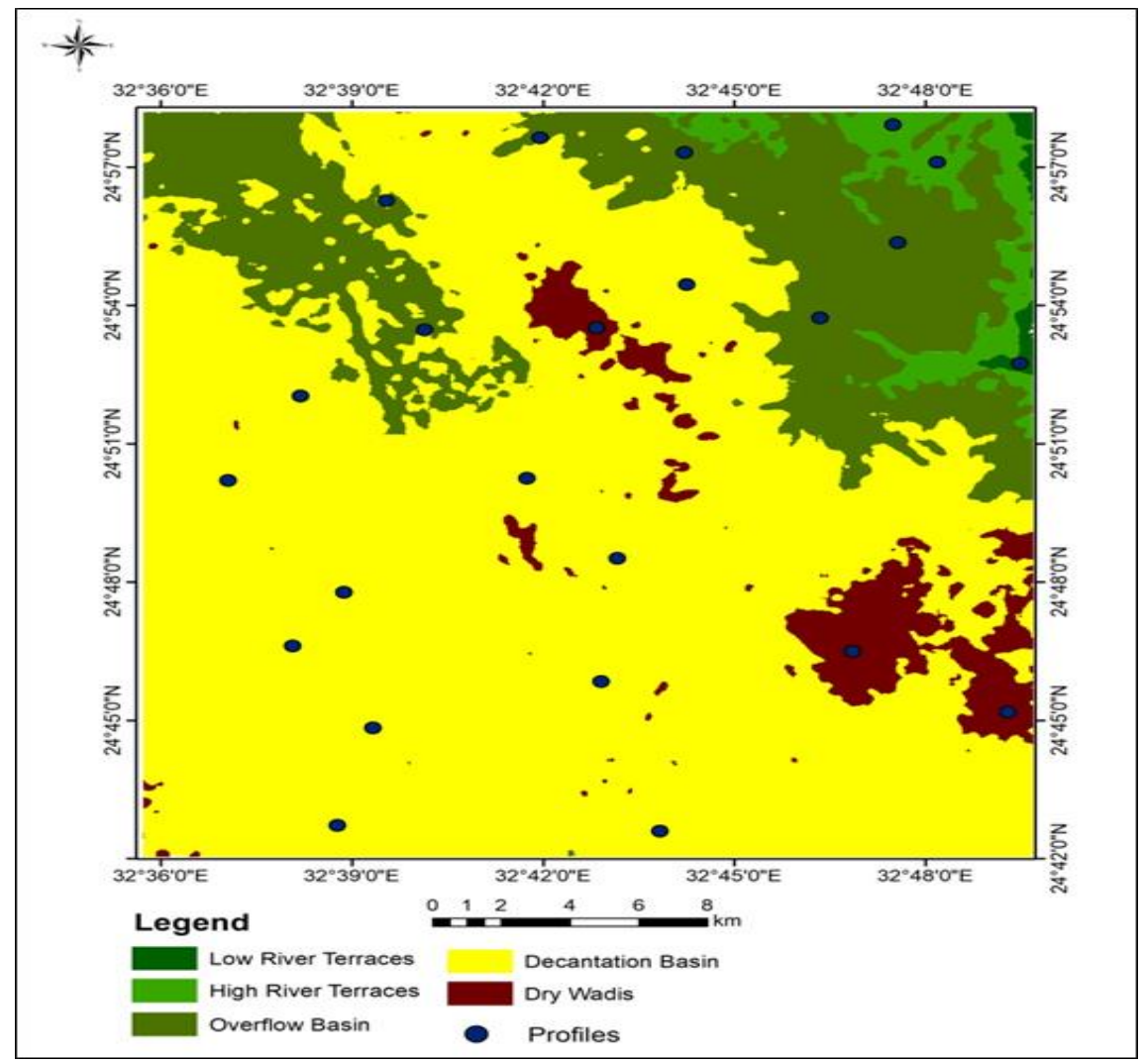

Fig. (2). Geomorphological map and soil profiles locations of the studied area.

Table (2). The geomorphological unit of the studied area.

\begin{tabular}{|c|l|c|c|}
\hline No. & Geomorphological Unit & Code & Area $\left(\mathrm{km}^{2}\right)$ \\
\hline 1 & River terraces ( High and Low) & RT & 26.013 \\
\hline 2 & Overflow basin & OB & 136.082 \\
\hline 3 & Decantation basin & DB & 504.23 \\
\hline 4 & Dry valleys & DV & 35.289 \\
\hline \multicolumn{2}{|c|}{ Total } & 701.62 \\
\hline
\end{tabular}

\section{Soils properties}

The characteristics of soil profiles representing the studied area are presented in Table (3). Data in Table (3) show that, the study area is almost flat (the slope ranged between 0.21 to $0.31 \%$ ) having deep soil profiles (>140 cm depth) with well drainage conditions. The soil texture varied from gravely sandy loam (GSL), sandy loam (SL), sandy clay loam (SCL) to loamy sand (LS). The soil reaction changed from slightly to moderately alkaline with $\mathrm{pH}$ values ranging between 7.8 to 8.8 . The soil salinity (electric conductivity, EC) differed from 3.5 to $161.9 \mathrm{dS} / \mathrm{m}$. This is might be due to salt accumulation in profiles and salty groundwater. Organic Matter content (OM) in these soils varied from 2.2 to $5.6 \%$, which might refer to organic residuals and organic matter manuring. Calcium carbonate $\left(\mathrm{CaCO}_{3}\right)$ and gypsum contents 


\begin{tabular}{|c|c|c|c|c|c|c|c|c|c|c|c|c|c|c|c|c|c|c|c|c|c|c|c|}
\hline 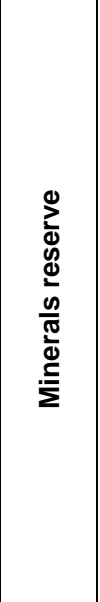 & 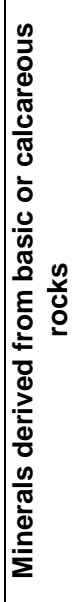 & 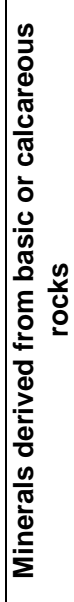 & 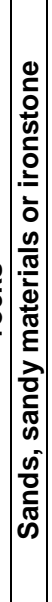 & 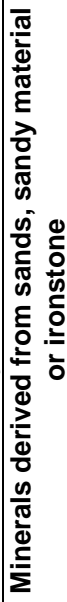 & 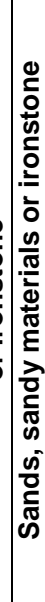 & 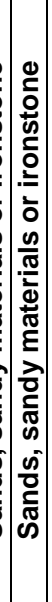 & 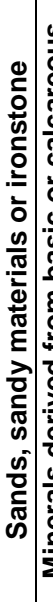 & 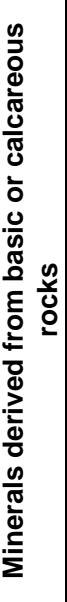 & 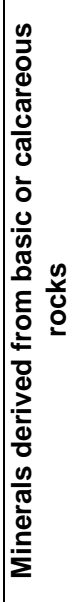 & 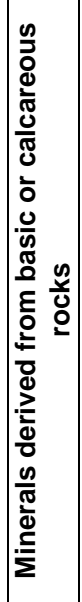 & 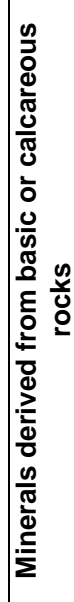 & 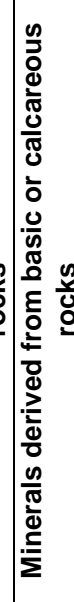 & 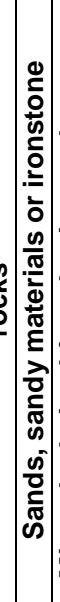 & 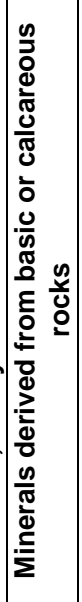 & 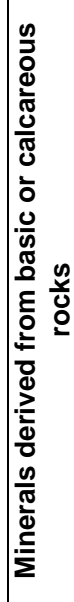 & 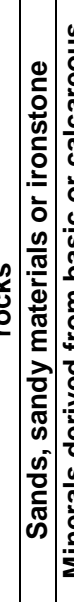 & 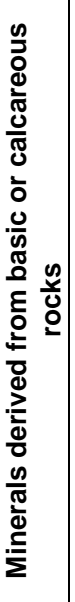 & 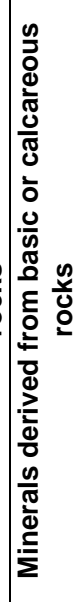 & 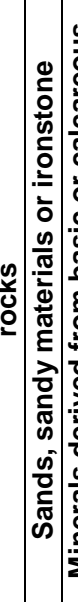 & 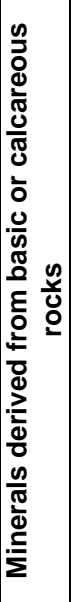 & 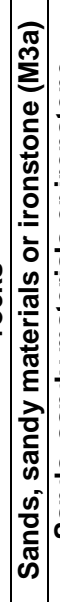 & 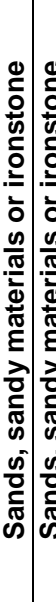 & 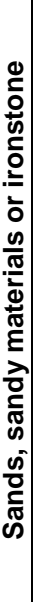 \\
\hline 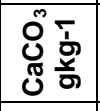 & ర్లి & ర్లి & ని & 우 & ని & & & q & ల్లి & டீ & $q$ & ల్లి & ని & 요 & R & ని & 웅 & ్․ & 으 & 8 & 의 & F & 우 \\
\hline ¿ू。 & 卢 & ஜூ & 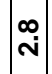 & ஸे & "ొ & $\begin{array}{l}0 \\
\dot{m}\end{array}$ & & $\bar{m}$ & ભm & $\hat{\mathbf{N}}$ & $\stackrel{m}{\text { in }}$ & $\stackrel{\sim}{N}$ & $\vec{t}$ & $\dot{m}$ & $\stackrel{m}{\text { ம் }}$ & $\mid \begin{array}{l}\mid \\
\mathscr{q}\end{array}$ & พี่ & $\stackrel{\bullet}{\oplus}$ & స̃ & \begin{tabular}{|l|}
0 \\
$\dot{m}$
\end{tabular} & $\begin{array}{l}0 \\
\omega \\
\omega\end{array}$ & $m$ & $\stackrel{m}{N}$ \\
\hline 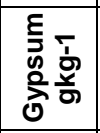 & $\mp$ & ْ̊ & $\begin{array}{l}0 \\
\infty \\
\infty\end{array}$ & 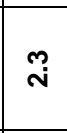 & ஜூ & $\mid \begin{array}{l}\infty \\
\dot{\gamma}\end{array}$ & (5) & $\bar{f}$ & L & $\hat{\emptyset}$ & 웅 & స్ల & $\mid \begin{array}{l}1 \\
\underline{0} \\
0\end{array}$ & $\stackrel{m}{9}$ & 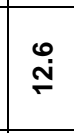 & $\ddot{\bullet}$ & $\stackrel{+}{\stackrel{\infty}{\circ}}$ & $\stackrel{\llcorner}{\longrightarrow}$ & $\stackrel{\infty}{+}$ & 㐌 & 象 & $\begin{array}{r}\hat{Q} \\
\dot{Q}\end{array}$ & فُ \\
\hline 密 & $\stackrel{\infty}{\leftarrow}$ & $\stackrel{9}{\leftarrow}$ & \pm & $\stackrel{9}{\stackrel{9}{2}}$ & $\bar{N}$ & $A$ & & $\stackrel{\infty}{\stackrel{\infty}{0}}$ & $\bar{N}$ & $\stackrel{\infty}{\sim}$ & $\stackrel{\infty}{\leftarrow}$ & $\stackrel{2}{2}$ & ని & $\bar{N}$ & 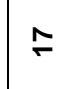 & $\stackrel{\infty}{=}$ & $\stackrel{9}{\stackrel{0}{0}}$ & ฉิ & กี & $\approx$ & 인 & & 요 \\
\hline 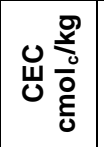 & $\stackrel{0}{\square}$ & $\widehat{\overbrace{}}$ & ò & ঞ் & 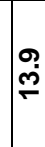 & & & 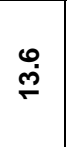 & $\stackrel{\infty}{\stackrel{\infty}{\mp}}$ & ్ㅗㅇ & ڤ̆ & $\stackrel{?}{\check{r}}$ & $\overline{\dot{f}}$ & $\stackrel{\nabla}{\sigma}$ & 吊 & 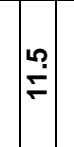 & $\stackrel{\infty}{\stackrel{\infty}{\sigma}}$ & ̃ & ஓे| & $\stackrel{m}{\sim}$ & $m$ & & 요 \\
\hline 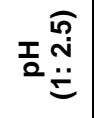 & $\stackrel{\infty}{\stackrel{\infty}{\sim}}$ & $\underset{\infty}{\infty}$ & $\infty$ & $\stackrel{L_{\infty}}{\infty}$ & $\underset{\infty}{\infty}$ & $|\bar{\infty}|$ & & ఎ & $\infty$ & $\infty$ & $\infty$ & 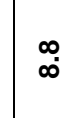 & $\bar{\infty}$ & $m$ & $\stackrel{\infty}{\Gamma}$ & $\infty$ & $\stackrel{\infty}{N}$ & $\bar{\infty}$ & $\mid$ & $\bar{\infty}$ & $\stackrel{\sigma}{1}$ & & 趈 \\
\hline 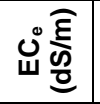 & 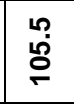 & ลั. & $\hat{\dot{\sigma}}$ & 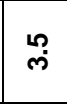 & $\stackrel{\mathscr{0}}{\circ}$ & 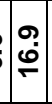 & 占 & 근 & $\stackrel{\infty}{\rightarrow}$ & \begin{tabular}{l}
\multirow{2}{*}{} \\
$\dot{\sigma}$
\end{tabular} & $\stackrel{\overbrace{}}{\stackrel{\theta}{0}}$ & ஜூ & $\overline{\underline{\rho}}$ & ֻ̊ & 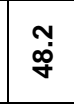 & $\left.\mid \begin{array}{l}m \\
0 \\
0 \\
0\end{array}\right]$ & î́ & $\stackrel{\mathfrak{N}}{\infty}$ & \begin{tabular}{|l|}
$\infty$ \\
$\infty$ \\
$\infty$
\end{tabular} & 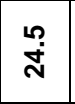 & : & & ब. \\
\hline 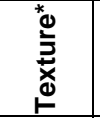 & ळ్ & $\vec{\omega}$ & है & क & कु & 岁 & & कु & ळ్ర & हु & $\stackrel{0}{0}$ & ळூ & $\mid \vec{\Phi}$ & ळூ & Фூ & $|\vec{\Phi}|$ & ల్ల & $\vec{\omega}$ & ळ్ & ग् & $\mid \vec{\omega}$ & & ๑ \\
\hline 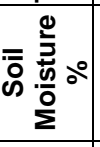 & 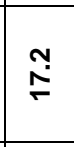 & 它 & $\stackrel{-}{\infty}$ & $\stackrel{\infty}{\stackrel{\infty}{\sim}}$ & $\stackrel{9}{\dot{f}}$ & & & $\stackrel{\infty}{\infty}$ & $\stackrel{\infty}{\infty}$ & 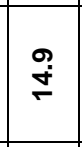 & $\stackrel{m}{\rho}$ & ஸึ & $\mid \begin{array}{l}0 \\
\dot{+}\end{array}$ & ्ְ? & $\begin{array}{l}\text { 号 } \\
\stackrel{\theta}{0}\end{array}$ & $\because$ & $\stackrel{\infty}{\stackrel{\infty}{+}}$ & 号 & 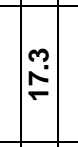 & 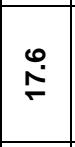 & $|\bar{\infty}|$ & & 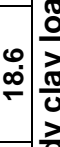 \\
\hline 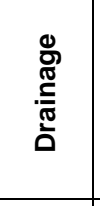 & 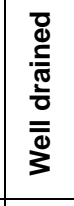 & 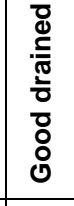 & 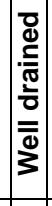 & 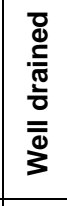 & 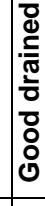 & 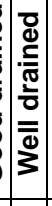 & & 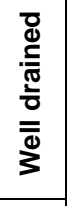 & 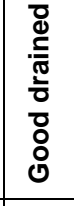 & 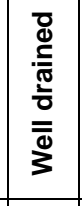 & 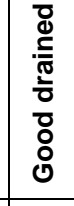 & 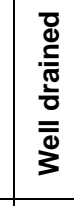 & 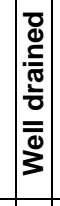 & 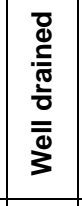 & 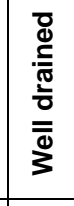 & 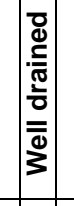 & 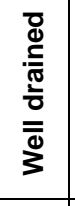 & 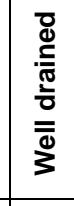 & 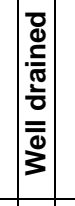 & 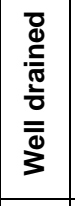 & 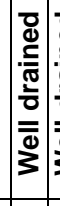 & 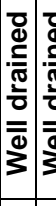 & 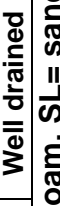 \\
\hline $\begin{array}{l}\frac{5}{\bar{t}} \\
\stackrel{0}{\Delta} \\
\end{array}$ & 윰 & 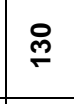 & ధొ & เి & f & ? & 욤 & 움 & $\vec{T}$ & $\stackrel{2}{2}$ & g & ํㅛㅁ & 으 & 욤 & ำ & 음 & ำ & 요 욤 & 욤 & 号 & 요 & & 노 \\
\hline $\begin{array}{l}\circ \\
\stackrel{\circ}{0} \\
\stackrel{\circ}{0} \\
\end{array}$ & ָָָ & స్తి & స్తి & গิ & हु, & $\begin{array}{l}m \\
0\end{array}$ & 赵 & న్ & సু & సָָ & స్త & ণ্ণ & $\mid \begin{array}{c}\text { ș } \\
\text { on }\end{array}$ & $\frac{\infty}{0}$ & స్ & $\mid \begin{array}{c}\mathbb{2} \\
\mathfrak{o} \\
\mathbf{0}\end{array}$ & స్ & $\frac{9}{0}$ & $\mid \overline{y ্ t}$ & ஸ̃ & $\stackrel{N}{0}$ & $\frac{\alpha}{\alpha}$ & $\stackrel{\infty}{0}$ \\
\hline 浐 & $r$ & $N$ & m & $\nabla$ & in & & & $\infty$ & a & 우 & $\mp$ & $\simeq$ & $\stackrel{m}{=}$ & $\stackrel{+}{J}$ & 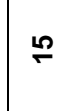 & $\stackrel{\varrho}{\bullet}$ & $\tilde{ح}$ & $\stackrel{\infty}{\leftarrow}$ & $\stackrel{9}{?}$ & ని & $\bar{N}$ & & న \\
\hline
\end{tabular}


varied from 10 to 300 and from 2.3 to $68 \mathrm{~g}$ kg-1, respectively (Table 3). Cation Exchange capacity (CEC) recorded moderate values of 8.8 to $19.8 \mathrm{cmol}_{\mathrm{c}} / \mathrm{kg}$ soil. These values refer to a moderate content of clay and organic materials. Exchangeable sodium percentage (ESP) ranged from 14 to 25 indicating sodic soils (> 15). The soil moisture content flocculated from 14.5 to $19.4 \%$. The investigated area covered by different minerals derived from basic or calcareous rocks and Sands, sandy materials or ironstone as shown in Table (3).

\section{Land productivity evaluation Riquier Land Productivity Index (RLPI)}

Riquier et al. (1970) described land productivity as the primary soil capability to product amount of crops per hectare in one year.

According to Riquier et al. (1970) and NRCS (2007), the results of Riquier Land Productivity Index of the investigated area presented in Table (4) and Fig. (3) showed two Productivity classes namely average and poor as follows:

Table (4). Riquier Land Productivity Index grades and classes of the studied area.

\begin{tabular}{|c|c|c|c|c|c|}
\hline No. & Mapping unit & $\begin{array}{c}\text { Riquier Land Productivity } \\
\text { Index RLPI (\%) }\end{array}$ & Grade & Class & Area / km \\
\hline 1 & Low River Terraces & 10.9 & IV & Poor & 2.59 \\
\hline 2 & High River Terraces & 18.219 & IV & Poor & 23.43 \\
\hline 3 & Overflow Basin & 17.26 & IV & Poor & 136.08 \\
\hline 4 & Decantation Basin & 23.34 & III & Average & 504.23 \\
\hline 5 & Dry Wadis & 17.442 & IV & Poor & 35.29 \\
\hline
\end{tabular}

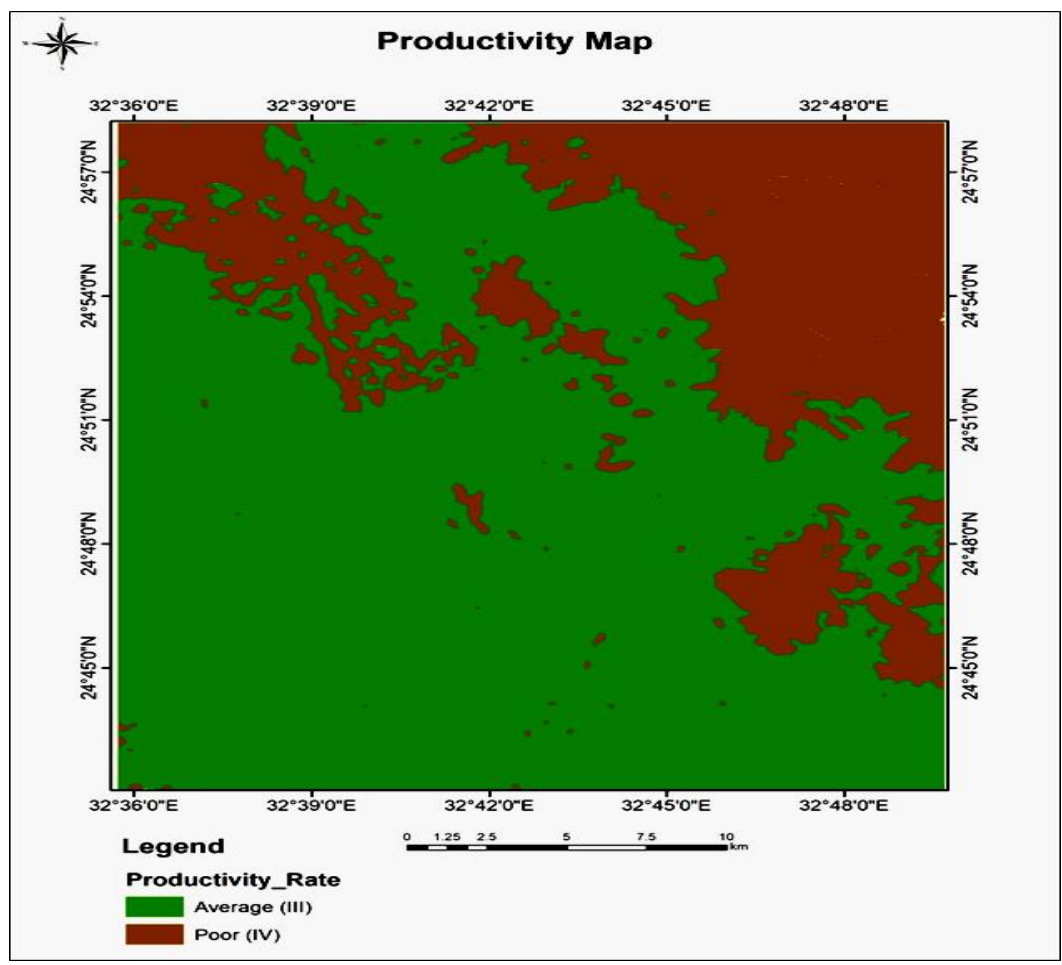

Fig. (3): Land productivity classes of the studied area. 


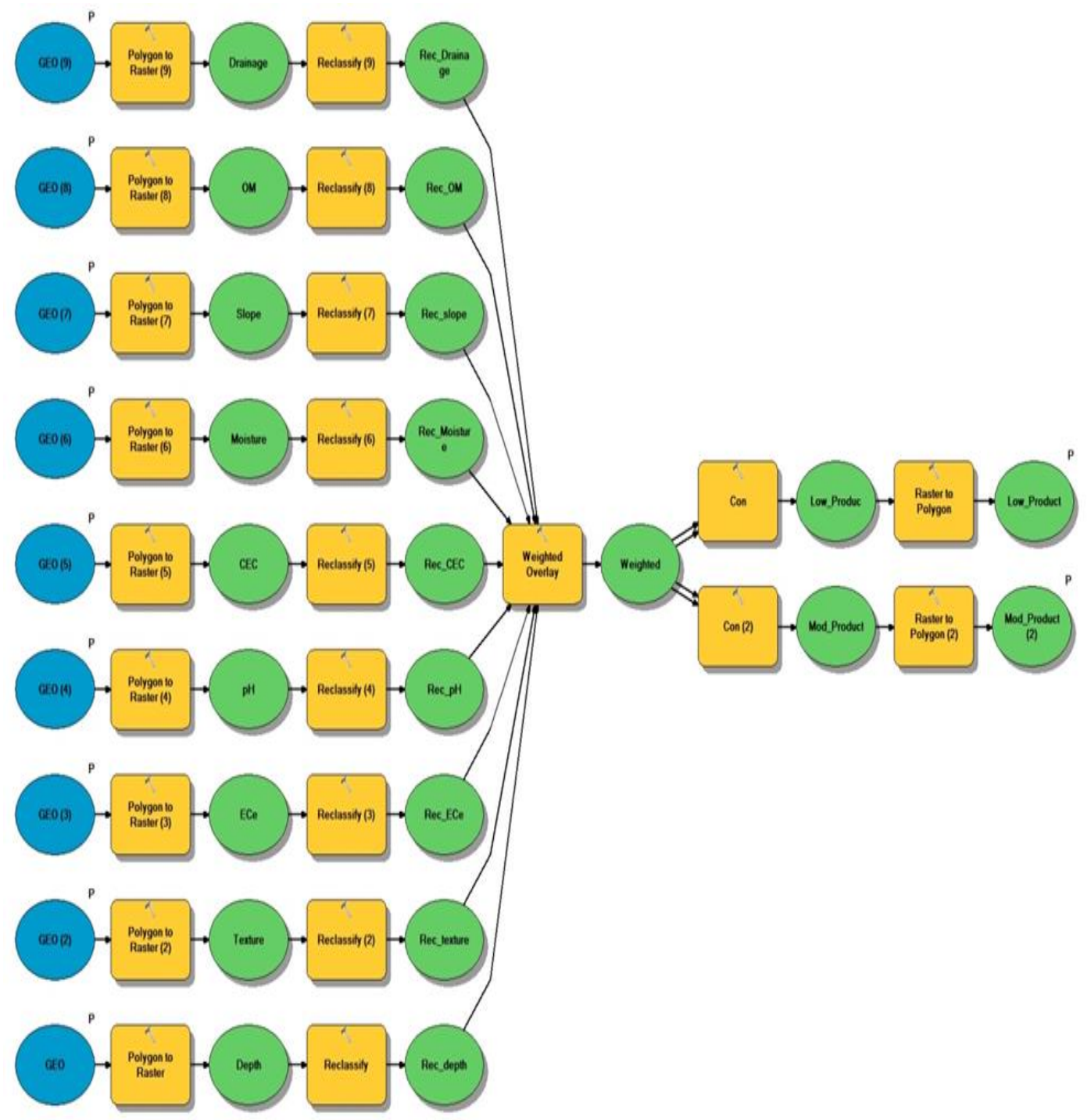

Fig. (4): Flowchart of the land Productivity spatial model.

1. Average Land Productivity class (grade III) covered an area of about 50422.9 hectares representing $71.87 \%$ of the studied area. These soils are fairly suited to general agricultural utilization with some restricted factors such as slopes; soil depths; permeability; soil textures; drainage; flood hazards; or fertility levels. All these factors might act alone or in combination.
2- Poor Land Productivity class (grade IV) covered an area of about 19738.49 hectares representing $28.13 \%$ of the studied area. These soils are inadequate for agricultural utilization. They are severely limited in their agricultural potential because of shallow soil depths; less permeable subsoil; steeper slope; or more clayey or gravelly surface soil textures than Grade 3 soils. These soils have also poor drainage; greater flood hazards; 
hummocky micro-relief; salinity; or fair to poor fertility levels. All these factors might act alone or in combination

\section{Land Productivity Spatial Model (LPSM)}

Land productivity spatial model was examined to determine the land productivity classes of the studied area. Identifying land productivity is essential to preparation a well management plan for the use of natural resources belongs to this promising vital region. Soil properties were subjected to laboratory analyses and office works then they were weighted and ranked to identifying land productivity. This model includes all the effective soil properties (Fig. 4) that required for productivity mapping according to specific criteria (soil depth, organic matter, texture, soluble salt, soil reaction, cation exchange capacity, drainage, slope, moisture content).

The produced productivity map as a result of implementing land productivity spatial model recognized two classes, moderate and low productivity map as follows:

\section{Moderate productivity class}

This class occupies an area of about $542.31 \mathrm{~km}^{2}$ that representing $77.29 \%$ of the studied area (Fig. 5). This area suffers from some limiting factors that impede growing some crops. Therefore, it needs special conservation practices. This area can be managed with some effort to be suitable for agricultural utilization.

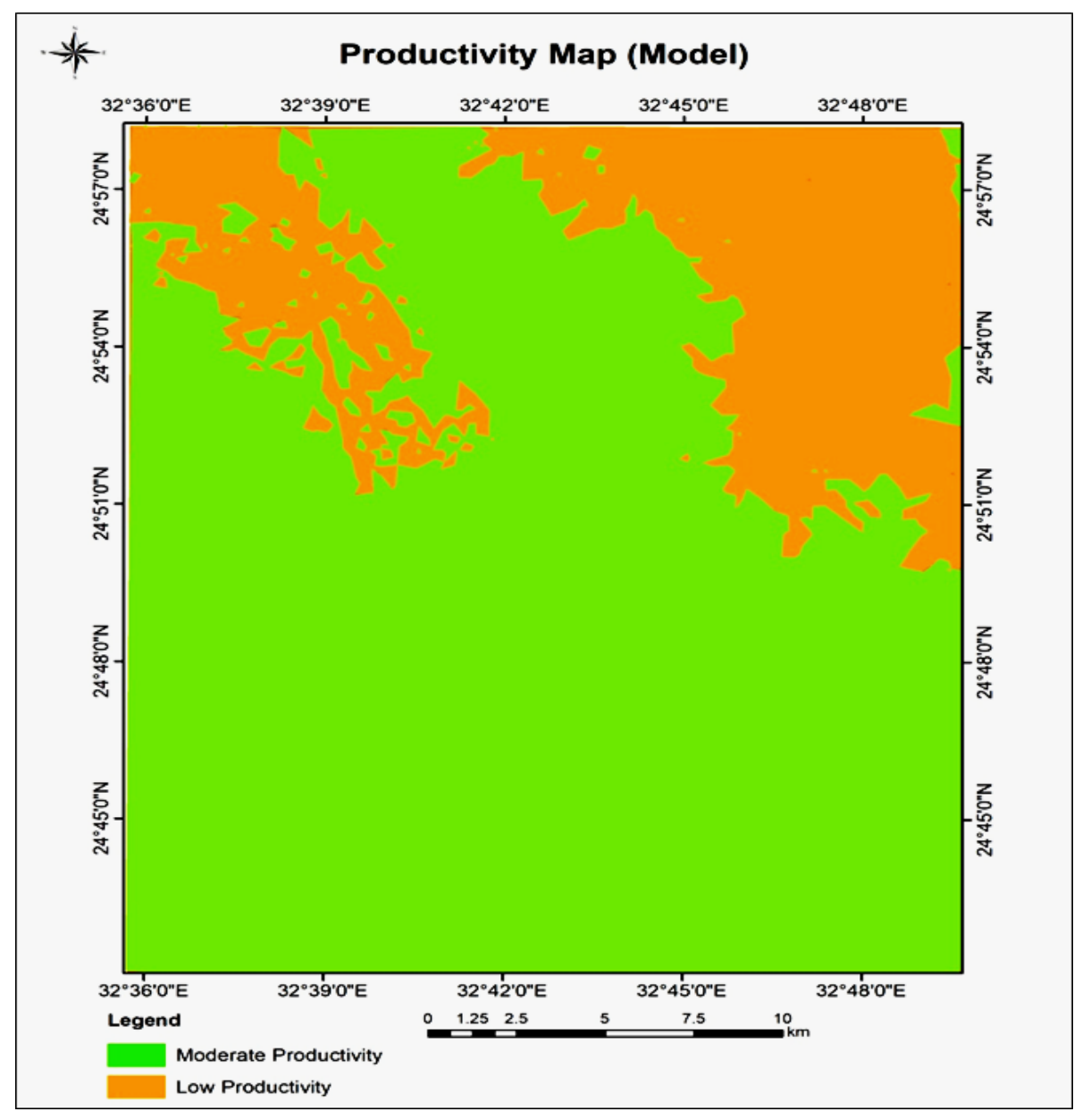

Fig. (5): Productivity map based on the spatial model 


\section{2- Low productivity class}

This class occupies an area of about $159.31 \mathrm{~km}^{2}$ that representing $22.71 \%$ of the studied area (Fig. 5). This area has a very severe limitations that restrict their use for agriculture propose since its agriculture productivity is low to marginal. Therefore, this area might be served for forage crops and agro-forestry systems.
A significant correlation coefficient was observed between the Land Productivity Spatial Model (LPSM) and Riquier Land Productivity index (RLPI). The obtained correlation coefficient for some soil properties of land productivity are shown in Table (5) and Fig. (6).

Table (5). Land productivity classes and areas based on the LPSM and RLPI models.

\begin{tabular}{|c|c|c|}
\hline Capability class & LPSM model areas / $\mathbf{k m}^{2}$ & RLPI model areas $/ \mathrm{km}^{2}$ \\
\hline Moderate & 542.31 & ----------- \\
\hline Average & ------------ & 504.23 \\
\hline Low & 159.31 & --------- \\
\hline Poor & ---------- & 197.38 \\
\hline
\end{tabular}

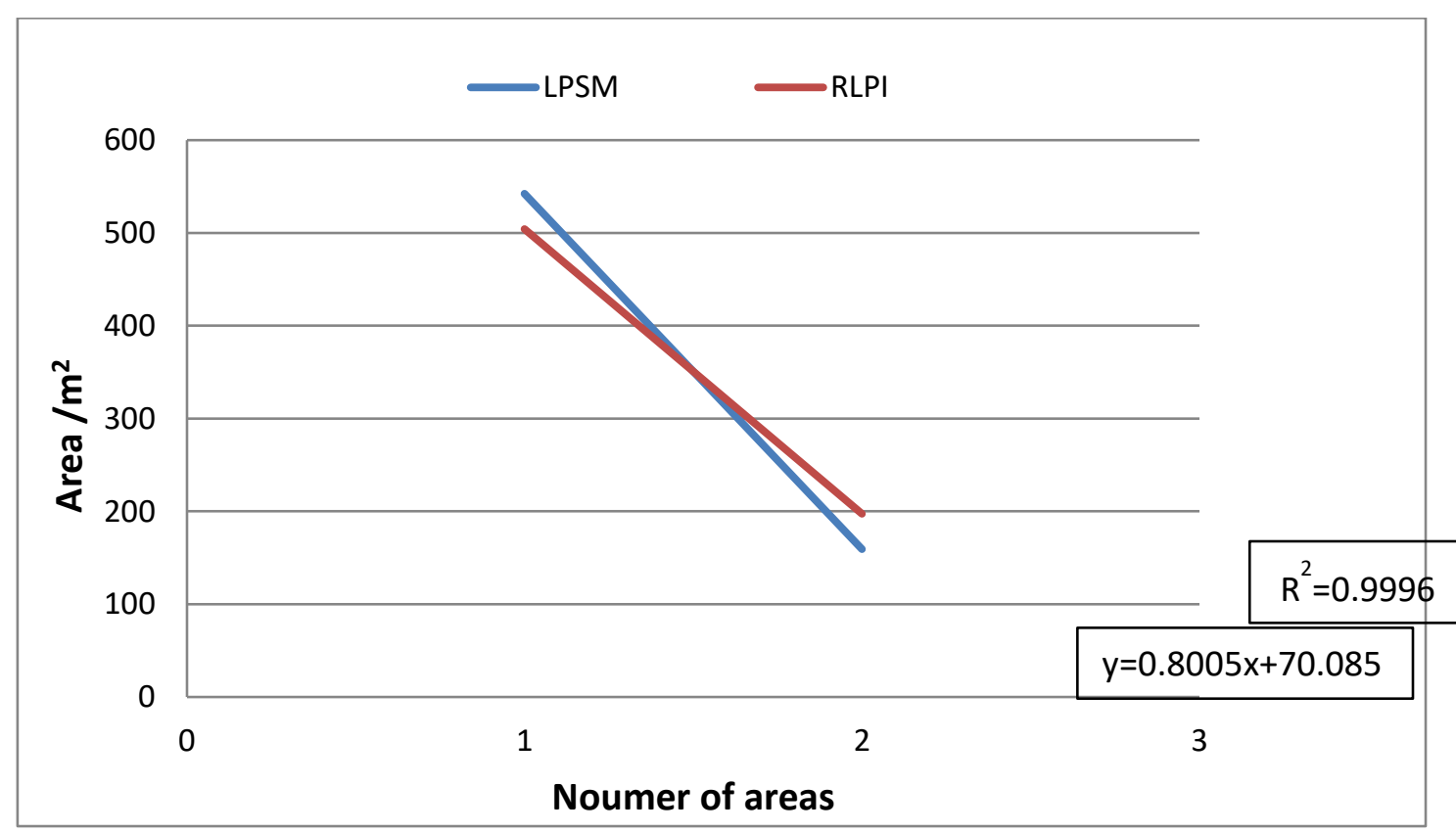

Fig. (6): Correlation between productivity areas based on the LPSM and RLPI models.

\section{Conclusion}

The studied area belongs to two land productivity classes with Riquier Land Productivity Index (RLPI), grade III (average) and grade IV (poor) representing 71.87 and $28.13 \%$ of the total area respectively. Soils belong to two land productivity classes with Land Productivity Spatial Model (LPSM), moderate productivity and low 
productivity representing $\mathbf{7 7 . 2 9}$ and $22.71 \%$ of the total investigated area respectively. A significant correlation was observed between the Land Productivity Spatial Model (LPSM) and Riquier Land Productivity Index (RLPI). These lands have moderate to very severe limitations that restrict their use for agriculture sector and require special conservation practices. These soils have fair to marginal productivity and recommended for producing forage crops and agroforestry systems.

\section{REFERENCES}

Andzo-Bika, H.L. W. and E. Kamitewoko (2004). Role of agriculture in economic development of developing countries: case study of China and Sub-Saharan Africa (SSA). J. Agric. Soc. Res. 4 (2).

Baskan, O., O. Dengiz and I.T. Demirag (2017). The land productivity dynamics trend as a tool for land degradation assessment in a dry land ecosystem. Environ. Monit. Assess. 189: 212.

Burt, Rebecea Ed. (2014). Soil Survey Field and laboratory methods manual. Soil Survey Investigation Report No. 51, Ver. 2.0, Kellegg Soil Survey Laboratory, National Soil Survey Center, Natural Resources Conservation Service, USDA, Lincoln, Nebraska.

Constanza, R., B.G. Norton and B.D. Haskell (1992). Ecosystem health: new goals for environmental management. Island Press, Washington, DC.

Deng, X.Z., Q.O. Jiang and X. Wen (2011). Computer- Based Estimation System for Land Productivity. In: Lin, S., Huang, X. (Ed.), Advanced Research on Computer Education, Simulation and Modeling, Pt. I, pp. 317-321.

Dengiz, O. and M. Sağlam (2012). Determination of land productivity index based on parametric approach using GIS technique. Eurasian J. Soil Sci.1: 51-57.

Egyptian Meteorological Authority (2011). The normal's for Aswan station, (19602010), Ministry of Civil Aviation, Cairo, Egypt.

ESRI (2014). Arc Map version 10.2.2. User Manual, 380 New York Street, Redlands, California, 92373-8100, USA.

FAO (2007). Land Evaluation, towards a revised framework. FAO, Rome, Italy.

Field, D.J. (2017). Soil Security: Dimensions. Global Soil Security. Springer, pp. 15-23.

Fuglie, K.O., M. James and B. Eldon (2007). Productivity growth in U.S. agriculture. EB-9, U.S. Dept. of Agriculture (USDA), Econ. Res. Serv. USA.

ITT (2017). ITT Corporation, Westchester Avenue, White Plains, NY 10604, USA.

NRCS (2007). National Soil Survey Handbook. Title 430-VI. United States Department of Agriculture Natural Resources Conservation Service (USDA NRCS).USDA Web site. http://soils.usda.gov/technical/handbo ok/.

Osuji, E.E. and A. Henri-Ukoha (2017). Land productivity of different use levels of sustainable soil management techniques of arable crop farmers in Imo state, Nigeria, Int'l journal of agric. and rural dev. Saatfuto, Volume 20(2): 3029-3036.

Pearce, D. and J. Warford (1993). World without End. World Bank, Washington, DC.

Riquier, J., D.L. Bramao and J.P. Cornet (1970). A new system of soil appraisal in terms of actual and potential productivity. FAO, Soil Resources, Development and Conservation Service, Land and Water Development Division. FAO, Rome. 
Schartzl, R. and S. Anderson (2006). Basic concepts of soil organisms: genesis and morphology. Cambridge University Press. 96-100.

Soil Survey Staff (2014). Keys to soil taxonomy.12 ${ }^{\text {th }}$ ed. United States Department of Agriculture, Natural Resources Conservation Service, Washington, DC.

World Bank (2007). World development report: Agriculture for Development. Washington, DC. World Bank. Doi: 10.1596/978-0-8213-6807-7. ISBN 9780-8213-6807-7.
Zeydan, A.B. (2005). The Nile Delta in a global vision. Ninth International Water Technology Conference, IWTC9 2005, Sharm El Sheikh, Egypt.

Zhou, Q., W. Wu and H.B. Liu (2012). Evaluation of Cultivated Land Productivity of Wanyuan County, Sichuan Province Using GIS. Advanced Technology in TeachingProceedings of the 2009 3rd International Conference on Teaching and Computational Science (WTCS 2009). Springer, pp. 919-926. 


\section{تقييم إنتاجية الأراضي لمنطقة إدفو - أسوان - مصر باستخدام تقنيات الاستشعار من بعد ونظم المعلومات الجغرافية}

$$
\begin{aligned}
& \text { احمد ابراهيم الاسوقي، ياسر عبد العال سيد } \\
& \text { قسم علوم الأراضي والمياه - كلية الزراعة- جامعة الأزهر - أسيوط ليرل }
\end{aligned}
$$

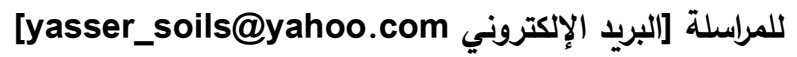

الملخص العربي

نتيجة للزيادة السكانية في مصر ونقص المساحات المزروعة كانت هناتك حاجة ماسة إلى استصلاح وزراعة العديد من

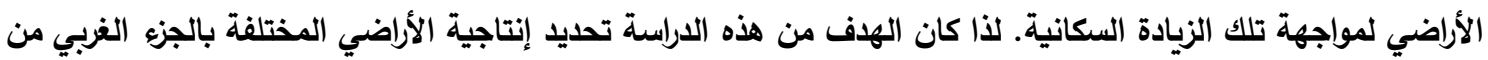
مدينة إدفو بمحافظة أسوان باستخدام تقنيات الاستشعار من بعد ونظم المعلومات الجغرافية لإنية.

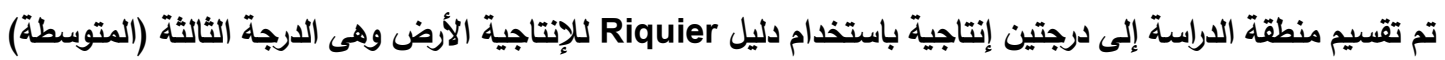
والدرجة الرابعة (الفقيرة) والتي تمثل 71,78\% و 28,13\% من من إجمالي المساحة المدروسة على التوالي، وأيضاً باستخدام النموذج المكاني للإنتاجية (LPSM) أظهرت هذه الأرض درجتين إنتاجية: أراضي متوسطة الإنتاجية وأراضي فقيرة الإلفية والإنتاجية

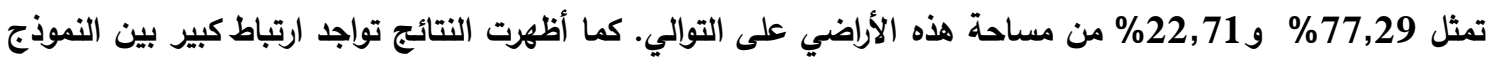
المكاني للإنتاجية Riquier لإنتاجية الأرض من خلال وجود معامل ارتباط لبعض خصائص الإض وإنتاجية

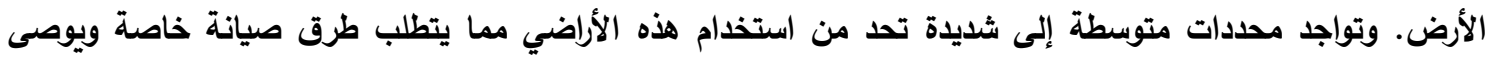
باستخدامها في زراعة بعض محاصيل العلف ونظم زراعة الغابات. 